\title{
Enfermedad por arañazo de gato. Características clínicas y de laboratorio en pacientes adultos hospitalizados por fiebre 0 adenopatías
}

\author{
G onzalo Eymin L, Antonio Zapata Pa, \\ Maricarmen Andrade A, Andrés Aizman S, \\ Luis Rojas 0 , Ricardo Rabagliati B. \\ Cat-scratch disease. \\ Review of eight adult patients \\ hospitalized for fever or adenopathy
}

\begin{abstract}
Background: Cat-scratch disease is common among children. Among adults the disease is less often considered in the differential diagnosis of enlarged lymph nodes and fever. Aim: To report the clinical and laboratory features of eight patients with cat-scratch disease. Material and methods: Review of the medical records of eight patients (aged 22 to 57 years, six males) with a serological diagnosis of cat-scratch disease (an IgG titer over 1:256, by immunofluorescence). Results: Only five patients recalled having had contact with cats. Seven had fever and weight loss. Six had excessive sweating and five had chills. Seven had painfully enlarged lymph nodes mainly in submandibular and axillary regions. All had an increased $\mathrm{C}$ reactive protein and six had elevated erythrocyte sedimentation rate. Five had leukocytosis and four an elevated serum lactate dehydrogenase. The disease subsided in all, even in one patient that did not receive antimicrobials. Conclusions: Cat-scratch disease should be considered in the differential diagnosis of adult patients with lymph adenitis and fever (Rev Méd Chile 2006; 134: 1243-48).
\end{abstract}

(Key w ords: Cat-scratch disease; Immunoglobulin G; Lymphadenitis)

Recibido el 3 de octubre, 2005. Aceptado el 4 de abril, 2006.

Departamento de Medicina Interna, Facultad de Medicina, Hospital Clínico de la Pontificia Universidad Católica de Chile. Santiago de Chile.

anterno de $7^{\circ}$ año de Medicina

$\mathrm{L}^{\mathrm{a}}$ a enfermedad por arañazo de gato es una enfermedad infecciosa, bacteriana, producida por Bartonella henselae, caracterizada, en la mayoría de los casos, por la aparición subaguda de una linfadenopatía regional autolimitada ${ }^{1}$. Fue

Correspondencia a: Gonzalo Eymin L Marcoleta 367, Santiago, Chile. Fax: 6394985. E mail: geymin@med.puc.cl descrita en 1950 en Francia, pero no fue sino hasta 1992 que Dolan y cols aislaron B henselae de linfonodos de pacientes afectados ${ }^{2,3}$. Afecta con mayor frecuencia a jóvenes menores de 20 años inmunocompetentes y la mayoría de las veces tiene un curso benigno y autolimitado ${ }^{4,5}$. En adultos, ya sea por lo pleomórfico de su presentación, o bien por la falta de reconocimiento clínico, 
no es raro que el diagnóstico se plantee luego de varias evaluaciones clínicas, exámenes de laboratorio y visitas a diferentes especialistas. Más de $90 \%$ de los pacientes tiene el antecedente de contacto con gatos, y entre $55 \%$ y $83 \%$ tiene el antecedente de arañazo de gato ${ }^{3,4,6}$. En este sentido, hay que considerar que existen reportes que plantean que las pulgas de los gatos puedan tener un rol importante en la transmisión de la enfermedad ${ }^{7-9}$

El presente trabajo describe las características clínicas de 8 pacientes adultos, con enfermedad por arañazo de gato diagnosticada en el Servicio de Medicina del Hospital Clínico de la Pontificia Universidad Católica de Chile.

\section{PACIENTES Y MÉTODOS}

El estudio se realizó en el Servicio de Medicina del Hospital Clínico de la Pontificia Universidad Católica de Chile, en la ciudad de Santiago. Este servicio consta de 56 camas, donde se hospitalizan pacientes mayores de 15 años por patología médica, del cual mensualmente egresan entre 250 y 300 pacientes.

Se buscaron, por medio de epicrisis, aquellos pacientes hospitalizados entre enero de $2001 \mathrm{y}$ septiembre de 2005, cuyo diagnóstico de egreso fuera enfermedad por arañazo de gato. Se identificaron 8 casos, a partir de los cuales se procedió a revisar la ficha clínica en forma retrospectiva, consignando los siguientes datos: sexo, edad, estudio pre-hospitalización, síntomas y su duración (fiebre, calofríos, diaforesis, baja de peso, etc.), antecedente de contacto o arañazo de gato, presencia de adenopatías, localización, número, sensibilidad, eritema de la zona, tamaño, supuración, temporalidad con el contacto o arañazo, duración, presencia de visceromegalias, lesiones cutáneas asociadas, alteraciones secundarias asociadas a la enfermedad (síndrome de Parinaud, encefalopatía, mielitis, radiculitis, osteomielitis, endocarditis), exámenes de laboratorio, imágenes, biopsias, diagnóstico diferencial inicial, el tiempo en llegar al diagnóstico en la hospitalización, uso de antibióticos previos al diagnóstico, tratamiento indicado y respuesta a éste.

\section{RESULTADOS}

Del total de pacientes, 6 eran de sexo masculino. La edad promedio fue $31 \pm 14$ años (22 a 57 años). Cinco pacientes recordaban haber tenido contacto con gatos. En cuanto a los síntomas, 7 pacientes presentaron fiebre, con un promedio de temperatura al ingreso de $37,6 \pm 0,97^{\circ} \mathrm{C}$, llegando hasta $38,6^{\circ}$ como temperatura máxima, 6 presentaron diaforesis, 5 calofríos y 7 baja de peso leve (1 a 4 $\mathrm{kg})$. La duración promedio de los síntomas fue de $13 \pm 8$ días (3-30). Cinco pacientes habían recibido antibióticos en forma ambulatoria, previo a la hospitalización (ciprofloxacino, cloxacilina, flucloxacilina, cefadroxilo).

En cuanto al examen físico, 7 pacientes presentaron adenopatías. De ellos, 3 en localización submandibular, 3 cervical, 2 supraclavicular, 2 inguinal, 1 axilar y 2 de ellos tenía más de una región ganglionar comprometida. En la totalidad de los casos se trataba de adenopatías sensibles al examen físico, 3 con eritema local y en ninguno de los casos hubo supuración. El tamaño promedio fue de $3,2 \mathrm{~cm}$, con un máximo de $7 \mathrm{~cm}$ y un mínimo de 1,5 cm (DS 2,5). Tres pacientes presentaron hepatoesplenomegalia, y en un caso se presentó exantema macular en tronco y extremidades.

La LDH estaba elevada en cuatro pacientes, en promedio $249 \pm 68 \mathrm{mg} / \mathrm{dl}$ (165 a 365). En el hemograma destacaba en 5 casos leucocitosis y en 2 desviación a izquierda. En un caso llamó la atención anemia normocítica normocrómica y en otro eosinofilia de 25\% (2.850 eosinófilos/ $\mathrm{mm}^{3}$ ). En 6 pacientes la VHS estaba elevada, con un promedio de $50 \pm 34 \mathrm{~mm} / \mathrm{h}$ (4 a 100) y los niveles de proteína $\mathrm{C}$ reactiva estaban elevados en la totalidad de los casos, con un promedio de $6,5 \pm 4,6 \mathrm{mg} / \mathrm{dl}(2$ a 11,3$)$. La mitad presentó SGOT discretamente elevada y en tres casos hubo aumento de las fosfatasas alcalinas. Ningún paciente presentó aumento de la bilirrubina. En todos los casos el diagnóstico fue confirmado con IgG por inmunofluorescencia en suero, con punto de corte de positividad en dilución $\geq 1: 256$.

Las hipótesis diagnósticas planteadas al ingreso fueron: en 4 pacientes adenitis supurada, en 3 enfermedad por arañazo de gato, en 2 síndrome 
Tabla 1. Características clínicas en 8 adultos con enfermedad por arañazo de gato

\begin{tabular}{|c|c|c|c|c|c|c|c|c|}
\hline $\begin{array}{l}\text { Hipótesis } \\
\text { principal } \\
\text { inicial }\end{array}$ & $\begin{array}{c}1 \\
\text { Mono } \\
\text { nucleosis }\end{array}$ & $\begin{array}{c}2 \\
\text { Linfoma }\end{array}$ & $\begin{array}{c}3 \\
\text { Linfoma }\end{array}$ & $\begin{array}{c}4 \\
\text { Adenitis }\end{array}$ & $\begin{array}{c}5 \\
\text { Síndrome } \\
\text { febril }\end{array}$ & $\begin{array}{c}6 \\
\text { Sindrome } \\
\text { febril }\end{array}$ & $\begin{array}{c}7 \\
\text { Enfermedad } \\
\text { arañazo } \\
\text { de gato }\end{array}$ & $\begin{array}{c}8 \\
\text { Adenitis }\end{array}$ \\
\hline Adenopatía & + & + & + & + & + & - & + & + \\
\hline Axilar & + & & & & & & & \\
\hline Cervical & + & + & + & & & & + & \\
\hline Submandibular & + & + & & & + & & & \\
\hline Yugular & + & & & & & & & \\
\hline Inguinal & & & & + & & & & + \\
\hline Supraclavicular & & + & & & & & & \\
\hline Tamaño cm & N/D & 1,5 & 2,5 & 7,0 & 2,0 & & 3,0 & 3,0 \\
\hline $\begin{array}{l}\text { Hepato } 0 \\
\text { esplenomegalia }\end{array}$ & + & & & & + & + & + & \\
\hline Rash & + & & - & & & & & \\
\hline
\end{tabular}

$\mathrm{N} / \mathrm{D}=$ no determinado.

Tabla 2. C aracterísticas de laboratorio en 8 adultos con enfermedad por arañazo de gato

\begin{tabular}{|c|c|c|c|c|c|c|c|c|}
\hline Hcto \% & 43,4 & 42,5 & 39 & 34,3 & 45,2 & 41,1 & 42,4 & 45,0 \\
\hline Blancos/ul & 5.200 & 11.400 & 9.200 & 12.400 & 8.200 & 11.900 & 13.300 & 14.000 \\
\hline Baciliformes \% & 5 & 1 & 2 & 0 & 12 & 12 & 0 & 2 \\
\hline Eosinófilos \% & 0 & 25 & 4 & 0 & 4 & 2 & 4 & 1 \\
\hline VHS mm/h & 4 & 96 & 42 & 98 & 54 & 75 & 50 & 16 \\
\hline PCR mg/dl & 6,9 & 3 & 2 & 3 & 8,8 & 11,3 & 14,7 & 2,7 \\
\hline SGOT mg/dl & 39 & 19 & 27 & 18 & 66 & 48 & 15 & 20 \\
\hline SGPT mg/dl & 68 & $\mathrm{~N} / \mathrm{D}$ & $\mathrm{N} / \mathrm{D}$ & $\mathrm{N} / \mathrm{D}$ & 104 & 40 & $\mathrm{~N} / \mathrm{D}$ & $\mathrm{N} / \mathrm{D}$ \\
\hline Fosfatasa alcalina U/dl & 89 & 58 & 90 & 119 & 208 & 129 & 50 & 88 \\
\hline GGT U/dl & 32 & $\mathrm{~N} / \mathrm{D}$ & $\mathrm{N} / \mathrm{D}$ & $\mathrm{N} / \mathrm{D}$ & 140 & 24 & $\mathrm{~N} / \mathrm{D}$ & $\mathrm{N} / \mathrm{D}$ \\
\hline
\end{tabular}

$\mathrm{N} / \mathrm{D}=$ no determinado.

linfoproliferativo, en 2 hepatitis, en 1 síndrome mononucleósico y en 1 adenitis tuberculosa.

El tiempo promedio en llegar al diagnóstico en el hospital fue de 3,6 $6 \pm 1,4$ días ( 2 a 5).

Siete pacientes recibieron azitromicina, $500 \mathrm{mg}$ por 5 a 7 días, y uno no recibió antibióticos. Todos los pacientes mejoraron con resolución rápida de la fiebre y más lenta de las adenopatías.

\section{DisCUSIÓN}

La enfermedad por arañazo de gato es una de las causas más frecuentes de adenopatía regional en niños y adultos. Es producida con mayor frecuencia por la Bartonella henselae, aunque puede ser producida por otras especies de Bartonella ${ }^{10}$. Un estudio en Estados Unidos de Norteamérica, que analizó 3 bases de datos, muestra que la incidencia en egresos hospitalarios de esta enfermedad es de 0,77 a 0,86 por 100.000 habitantes, siendo sólo $55 \%$ de éstos menores de 18 años.

Afecta principalmente a hombres menores de 20 años y la mayor incidencia de infección ocurre en otoño e inviemo ${ }^{4}$. Los criterios diagnósticos son: lesión dérmica u ocular primaria asociada a arañazo de gato (generalmente joven), linfoadenopatías en general vecinas a la lesión, pruebas de laboratorio 
que descarten otras etiologías, test intradérmico diagnóstico o test serológico positivo para $B$ henselae, biopsia del nódulo con hallazgos histopatológicos consistentes con enfermedad por arañazo de gato (bacilos teñidos con tinción argéntica de WarthinStary y linfadenitis granulomatosa supurada) ${ }^{2,3}$.

En general, ante un paciente que consulta por linfadenitis regional, el diagnóstico diferencial es amplio, debiendo contemplar entre las causas infecciosas la adenitis supurada por los siguientes agentes: Staphylococcus aureus, Streptococcus spp, abscesos cervicales de origen polimicrobiano, Mycobacterium tuberculosis y micobacterias atípicas, virus de inmunodeficiencia humana, virus de Epstein Barr, citomegalovirus, toxoplasma gondii, actinomyces spp. Además, debe tenerse en cuenta las neoplasias benignas y malignas de linfonodos, glándulas salivales y tiroides. Otras entidades como las enfermedades de Kimura, Kikuchi y Castleman, son infrecuentes y de origen desconocido aún, pero también deben considerarse en el diagnóstico diferencial ${ }^{11}$. Un estudio de 721 pacientes con masas cervicales mostró que $13,7 \%$ se debían a enfermedad por arañazo de gato, con un promedio de edad en este último grupo de 33 años ${ }^{12}$.

El cuadro clínico clásico se caracteriza por la presencia de una linfadenopatía regional, a veces acompañada de fiebre de semanas a meses de evolución y que comienza en relación a un arañazo de gato $2,13,14$. Es frecuente la presencia de una pápula roja en el sitio de inoculación, que puede preceder a la linfadenopatía en 10 a 14 días $^{14}$. Esta pápula dura de tres a diez días y evoluciona a una vesícula y, a veces, a nódulo, curando sin cicatriz ${ }^{2,13,15}$. En 5 a 10\% de los casos, el sitio de inoculación es el ojo o la mucosa oral (conjuntivitis no supurativa y úlcera oral, respectivamente). Las linfadenopatías pueden aparecer hasta 60 días después de la inoculación. Son habitualmente dolorosas, a veces con eritema de la piel y ocasionalmente supuradas (10\% a $15 \%)$, lo cual contrasta con nuestra serie, en que ningún paciente presentó adenopatías supuradas. El tamaño va de 1 a $5 \mathrm{~cm}$, pero pueden alcanzar los 10 $\mathrm{cm}$. Los grupos ganglionares más comprometidos son las axilas, epitróclea, cervicales, supraclaviculares, y submandibulares. En 85\% de los casos son únicas $^{16}$. En $2 / 3$ de los casos el compromiso es regional y en $1 / 3$ pueden comprometerse varios grupos ganglionares. Es raro el compromiso generalizado. Casi siempre se trata de adenopatías sensibles, lo cual las diferencia, en parte, de las adenopatías de los síndromes linfoproliferativos. Habitualmente se resuelven dentro de cuatro meses $^{17}$. En nuestra serie, el grupo más comprometido fue el submandibular y cerca de la mitad tenía más de un grupo ganglionar comprometido. Probablemente el compromiso axilar en nuestra serie fue menor, dado que es un grupo seleccionado de pacientes con enfermedad por arañazo de gato que llegan a hospitalizarse para estudio por adenopatías, en localizaciones poco familiares para los médicos y que, por lo mismo, plantean otros diagnósticos diferenciales.

La duración de los síntomas, en la mayoría de los casos, es mayor a una semana al momento de la consulta médica, lo cual se relaciona con lo relativamente bien tolerado de la enfermedad, situación que se repite en nuestra serie, en que la duración promedio fue de dos semanas.

En algunas personas la enfermedad se puede diseminar y puede comprometer hígado, bazo, ojos, etc. Los pacientes con enfermedad localizada, generalmente tienen un curso autolimitado, mientras que los con enfermedad diseminada pueden tener complicaciones mortales. De los pacientes presentados en nuestra serie clínica, no se observó ninguna manifestación de diseminación.

En nuestra serie, el síntoma más sensible fue la fiebre, la cual estaba presente en casi la totalidad de los casos. En segundo lugar están la diaforesis y la baja de peso, lo cual incide en que con frecuencia se estudie a estos pacientes con diagnóstico presuntivo de síndrome linfoproliferativo, tuberculosis u otros. Quizá el poco compromiso axilar influyó que en nuestra serie se retrasara el diagnóstico y que, por el contrario, haya sido una hipótesis diagnóstica inicial en sólo tres pacientes.

Un estudio retrospectivo en Francia analizó a 26 pacientes con diagnóstico de enfermedad por arañazo de gato y mostró que todos los pacientes presentaron adenopatías, y $46 \%$ las presentaron como único síntoma de la enfermedad; en todos se practicó estudio serológico y en 34\% la biopsia ganglionar fue la que llegó finalmente al diagnóstico ${ }^{18}$. En nuestra serie, un paciente se presentó sólo con síndrome febril y sin adenopatías, y en 
todos los casos las adenopatías se presentaron asociadas a fiebre, quizá reflejando que este signo pudo haber alertado a los médicos a pensar en diagnósticos más ominosos.

El diagnóstico serológico de elección hoy en día es la determinación de títulos de IgG mediante inmunofluorescencia. Títulos menores a 1:64 podrían representar infección antigua. Los pacientes con la enfermedad habitualmente tienen títulos iguales o mayores a 1:256. Títulos entre 1:64 y 1:256 se pueden encontrar en pacientes sanos (4$6 \%)$, y en el comienzo o final de la enfermedad. En este caso se recomienda repetir la prueba 10 a 14 días después, en busca de un aumento de los títulos. Títulos mayores a 1:256 son altamente sugerentes de infección. La sensibilidad y la especificidad del método va de $14 \%$ a $100 \%$ y de $40 \%$ a $100 \%$, respectivamente, dependiendo del antígeno usado y del punto de corte ${ }^{19}$. El cultivo es de muy baja sensibilidad, puesto que es un microorganismo de crecimiento fastidioso y muy difícil de aislar en tejidos. La histopatología es poco específica y sólo a veces se logra identificar el bacilo con tinción de Warthin-Starry. La detección por PCR aún está en estudio y no está estandarizada como prueba diagnóstica. En esta serie, todos los pacientes tuvieron títulos de 1:256, un cuadro clínico característico y mejoría con el uso de antibióticos (uno se mejoró sin antibióticos).

La mayoría de los pacientes tuvo una resolución gradual de los síntomas, incluso sin antibiótico. El único trabajo prospectivo, randomizado,

\section{REFERENCIAS}

1. Margileth A, Wear D, Hadfield T, Schlagel C, Sitgel G, Muhlbauer J. Cat-scratch disease. Bacteria in skin at the primary inoculation site. JAMA 1984; 252: 928-31.

2. Dolan M, Wong M, Regnery R, Jorgensen J, García M, Peters J et al. Syndrome of Rochalimaea henselae Adenitis Suggesting Cat Scratch Disease. Ann Intern Med 1993; 118: 331-6.

3. Fischer GW. The agent of cat scratch disease. In: Mandell G, Douglas R Jr, Bennett J; eds. Principles and Practice of Infectious Diseases. $3^{\text {rd }}$ ed. New York: Churchill Livingstone Inc., 1990; 1874-7. doble ciego, publicado por Bals y cols. avala el uso de azitromicina, $500 \mathrm{mg}$ el primer día, seguido de $250 \mathrm{mg}$ por cuatro días. De los 14 pacientes tratados con azitromicina, siete experimentaron una regresión de más de $80 \%$ del tamaño de la adenopatía en un mes, en comparación a 1 de 15 en el grupo placebo ${ }^{20}$. Sin embargo, este estudio no mostró mejoría en otros resultados clínicos, ni tampoco prevención de la diseminación ni aparición de complicaciones (endocarditis, encefalitis), por lo que algunos autores plantean que la recomendación de tratar a los pacientes inmunocompetentes sería cuestionable. Otros esquemas ensayados con éxito incluyen rifampicina, ciprofloxacina, gentamicina y cotrimoxazol ${ }^{20}$. En pacientes con riesgo vital, se recomienda el uso de azitromicina o gentamicina intravenosa, continuando con antibióticos orales, una vez estable, hasta completar 10 a 14 días.

En conclusión, la enfermedad por arañazo de gato debe estar siempre en la mente de un médico al enfrentarse a un paciente que consulta por adenopatías, e incluso debe considerarla como parte del diagnóstico diferencial del síndrome febril. Debe buscarse dirigidamente el antecedente epidemiológico de contacto con mascotas, en especial gatos. Sólo un alto índice de sospecha podría hacer que éste sea un diagnóstico ambulatorio, o en su defecto se realice precozmente dentro de la hospitalización, de modo que se eviten todos los gastos y procedimientos innecesarios en que se incurre con frecuencia.

4. Zangwill $K$, Hamilton D, Perkins B, Regnery $R$, Plikaytis B, Hadier J et al. Cat-Scratch disease in Connecticut: Epidemiology, risk factors, and evaluation of a new diagnostic test. $\mathrm{N}$ Engl J Med 1993; 329: 8-13.

5. Jackson L, Perkins B, Wenger J. Cat-scratch disease in the United States: an analysis of three national databases. Am J Public Health 1993; 83: 1707-11.

6. MARGILETH A. Cat-scratch disease: nonbacterial regional lymphadenitis; The study of 145 patients and a review of the literature. Pediatrics 1968; 42: 803-18.

7. Adal K, Cockerell C, Petri W. Cat-Scratch Disease, Bacillary Angiomatosis, and Other Infections Due 
to Rochalimaea. N Engl J Med 1994; 330: 1509-15.

8. Margileth A, Andrew M, Hayden G. Cat-Scratch Disease - From Feline Affection to Human Infection. N Engl J Med 1993; 329: 53-4.

9. Breitschwerdt EB, Kordick DL Bartonella Infection in Animals: Carriership, Reservoir Potential, Pathogenicity, and Zoonotic Potential for Human Infection. Clin Microbiol Rev 2000;13: 428-38.

10. Giladi M, Avidor B, Kietter Y, Abulafia S, Siater LN, WeLCH DF ET AL. Cat-scratch disease: The rare rol of Afipia felis. J Clin Microbiol 1998; 36: 2499-502.

11. Schwetschenau E, KeLley DJ. The adult neck mass. Am Fam Physician 2002; 66: 831-8.

12. Rddier GJ, Boedeker CC, Technau-Ihung K, SANder A. Cat-scratch disease: Otolaryngologic manifestations and management. Otolaryngol Head Neck Surg 2005; 132: 353-8.

13. CaRITHERS HA. Cat-scratch disease: An overview based on a study of 1200 patients. Am J Dis Child 1985; 139: 1124.

14. Margileth AW, Wear DJ, Hadfield TL, Schlagel CJ, Sitgel GT, Muhlbauer JE. Cat-scratch disease: bacteria in skin at the primary inoculation site. JAMA 1984; 252: 928-31.
15. Moriarty RA, Margileth AM. Cat-scratch disease. Infect Dis Clin North Am 1987; 1: 575-90.

16. Ladrón de Guevara D, Lobo G, Miranda M, Wu E, MuÑoz MA, Pérez A et al. Forma atípica de enfermedad por arañazo de gato: Compromiso óseo en dos pacientes pediátricos detectado mediante cintigrafía ósea. Revisión de la literatura. Rev Chil Infect 2003; 20: 202-9.

17. Rolain JM, Brouqui P, Koenler JE. Recommendations for Treatment of Human Infections Caused by Bartonella Species. Antimicrob Agents Chemother 2004; 48: 1921-33.

18. SANDER A, BeRnER R, Ruess M. Serodiagnosis of CatScratch Disease: Response to Bartonella Henselae in Children and a Review of Diagnostic Methods. Eur J Clin Microbiol Infect Dis 2001; 20: 392-401.

19. Bass J, Freitas B, Freitas A. Prospective randomized double-blind placebo-controlled evaluation of azithromycin for treatment of cat-scratch disease. Pediatr Infect Dis J 1998; 17: 447-52.

20. Margileth A. Antibiotic therapy for cat-scratch disease: Clinical study of therapeutic outcome in 268 patients and a review of the literature. Pediatr Infect Dis J 1992; 11: 474. 